\title{
Original
}

\section{Seguimiento a largo plazo de la endopielotomía anterógrada. Factores que influyen en el resultado}

\author{
Jose H. Amón Sesmero, Noelia del Valle González, Luis A. Rodríguez Toves, \\ Consuelo Conde Redondo, Verónica Rodríguez Tesedo, José M. Martínez-Sagarra Oceja \\ Servicio de Urología. Hospital Universitario Río Hortega. Valladolid.
}

\begin{abstract}
Resumen
Objetivo: Conocer los resultados de la endopielotomía con un seguimiento a largo plazo y determinar las variables que influyen en los mismos.

Material y Métodos: Se revisan los resultados de la endopielotomía anterógrada realizada en 77 pacientes con obstrucción de la unión pieloureteral (OUPU) con un seguimiento superior a 10 años. Se utilizó la curva de Kaplan-Meier para conocer la probabilidad de fracaso en un determinado momento.

Se realizó planimetría del área pielocalicial y se estudio su factor de forma para conocer la influencia de la hidronefrosis en el resultado. Se estudiaron otras variables como el grado de función renal, cirugía previa, coexistencia de litiasis y malformación renal asociada.

Resultados: El seguimiento medio fue de 149,26 meses. La probabilidad de fracaso fue de 26,9\%, 34,5\% y 36,8\% al año, 5 años y 10 años respectivamente. La mayor concentración de fracasos se produjo dentro de los 20 primeros meses.

El área pielocalicial media en los éxitos fue de 19,70 $\pm 8,32 \mathrm{~cm}^{2}$ vs $30,19 \pm 11,07 \mathrm{~cm}^{2}$ en los fracasos, ( $\mathrm{p}=0,018$ ).No hubo diferencias entre los valores del factor de forma en los éxitos y en los fracasos $(0,87$ vs $0,88, p=0,135$, respectivamente) La función renal $(45,1 \%$ en éxitos vs $40,9 \%$ en fracasos, $\mathrm{p}=0,625)$, la cirugía previa (62\% de éxito con cirugía previa vs $64,7 \%$ en primarias, $p=0,843)$, y la asociación con litiasis $(69,3 \%$ éxito con litiasis vs $61,1 \%$ sin litiasis, $p=0,541)$ no influyeron el resultado. La concomitancia de hidronefrosis y malformación renal afectó negativamente el resultado.

Conclusión: La tasa de éxito de la endopielotomía disminuye en seguimientos a largo plazo, si bien a partir del quinto año se mantiene estable. Seleccionar casos para esta técnica de acuerdo al área de la hidronefrosis podría mejorar el resultado.
\end{abstract}

Palabras clave: Endopielotomia. Resultados a largo plazo. Hydronephrosis area value.

\section{Long-term follow-up of antegrade endopyelotomy. Factors that influence the outcome}

\section{Abstract}

Objective: To find out the outcomes of endopyelotomy alter a long-term follow-up and determine the variables that may influence the results.

Material and Methods: We review the results obtained in 77 patients that underwent antegrade endopyelotomy with ureteropelvic junction obstruction, after 10- year follow-up. We used the Kaplan-Meier curve in order to determine the probable failure rate at a certain point. We took measurements of the pyelocalix area and studied its shape to find out the influence of hydronephrosis in the outcomes. Other variables, such as renal function, previous surgery, lithiasis and renal malformation associated, were analysed.

Results: Mean follow-up was 149.26 months. Faliure rate probability was $26.9,34.5$ and $36.8 \%$ a year, 5 years and 10 years later, respectively. Major failure concentration occurred in the first 20 months.

Mean pyelocalix area success was $19.70 \pm 8.32 \mathrm{~cm}^{2}$ vs $30.19 \pm 11.07 \mathrm{~cm}^{2}$ of failure, $(\mathrm{p}=0,018)$. There were no differences between the values of the shape factor in either success and failure. $(0,87$ vs $0.88, p=0.135$, respectively).

Renal function ( $45.1 \%$ success vs $40,9 \%$ failure, $\mathrm{p}=0,625)$, previous surgery (62\% success after previous surgery vs $64.7 \%$ first procedure, $\mathrm{p}=0.843)$, and lithiasis associated $(69.3 \%$ success through lithiasis vs $61.1 \%$ without lithiasis, $\mathrm{p}=0.541)$ did not affect the outcomes. Concomitance of hydronephrosis and renal malformation affected the outcomes negatively.

Conclusion: Endopyelotomy success rate reduces long-term follow-up, however, after the fifth year it becomes stable. Selecting cases to apply this technique according to the value of hydronephrosis area could improve the results. 
$\mathrm{D}_{1}$ esde su descripción por Wickham y Kellet $^{1}$ en 1983, la endopielotomía ha sido el tratamiento inicial de la obstrucción de la unión pieloureteral (OUPU) dada su escasa morbilidad, sencillez técnica y corto postoperatorio, a pesar de no alcanzar las tasas de éxito de la pieloplastia abierta ${ }^{2}$.

Dos hechos están cuestionando actualmente la vigencia de esta técnica: el primero, la aparición de otra opción de tratamiento mínimamente invasor como es la pieloplastia laparoscópica ${ }^{3}$, que reproduciendo los principios quirúrgicos de la pieloplastia abierta consigue unas tasas de éxito similares a ésta con las ventajas que aporta la cirugía laparoscópica; y el segundo, la observación de una caída en su tasa de éxito en seguimientos a largo plazo ${ }^{4,5,6 .}$

Presentamos en este trabajo el seguimiento a largo plazo de la endopielotomía en nuestra serie inicial de pacientes, antes de la introducción en nuestro servicio de la pieloplastia laparoscópica, y la identificación de los factores que han influido en los resultados de cara a conseguir mejorar éstos mediante una mejor selección de pacientes.

\section{MATERIAL Y MÉTODOS}

Revisamos las historias de 77 pacientes a los que se realizó una endopielotomía anterógrada por OUPU desde junio 1989 a febrero de 2001, (periodo anterior a la introducción de la pieloplastia laparoscópica en nuestro servicio, por tanto el estudio corresponde a pacientes no seleccionados). Sólo fueron excluidos los casos con función renal inferior al 20\% y estenosis excesivamente largas, no así, la sospecha de vaso cruzado. En la Tabla 1 están expresadas las variables demográficas de los pacientes y en la Tabla 2 las variables morfológicas y cualitativas.

El diagnóstico de OUPU se hizo en base a la clínica, urografía (UIV) y renograma isotópico diurético (RID).

Técnica

En esta serie de pacientes la endopielotomía anterógrada fue realizada bajo anestesia general con el paciente en posición prona (actualmente empleamos el decúbito dorsal) Previamente se realizó

Tabla 2 cateterismo retrógrado en posición de litotomía con el fin de opacificar vía excretora y valorar la longitud de la estenosis. EL abordaje renal fue realizado preferentemente por el cáliz medio. La dilatación del tracto de nefrostomía se llevó a cabo con catéter balón (Nefromax 30 Fr. Boston Scientific). Como hemos descrito con anterioridad ${ }^{7}$, antes de realizar la pielolisis, sustituimos el catéter ureteral por un doble $\mathrm{J}$ calibre 7 /8 Fr. por vía anterógrada. Con un resector montado con cuchillete de Collings incidimos la pelvis en dirección posterolateral, para continuar después el corte de la pared ureteral, de forma transpiélica en sentido caudal unos dos centímetros. Mantenemos el catéter doble $\mathrm{J}$ tutor durante 8 semanas.

Todos los pacientes fueron revisados a los 3 y 12 meses, y posteriormente con periodicidad anual. En cada visita se realizó una valoración clínica, analítica y se comprobó el resultado funcional mediante

\section{Tabla 1}

Edad media: 34,1 años. (r:9-73)

Sexo: 46 varones/21 hembras.

Lado: Derecho: 32 casos/Izquierdo: 35 casos.

Cirugía previa: Primarias 50 casos/Secundaria 17 casos Litiasis asociada: 13 casos.

Malformación asociada: 4 casos (riñón en herradura)

\begin{tabular}{lccc}
\hline Variables morfológicas & Éxito & Fracaso & valor $\boldsymbol{p}$ \\
\hline $\begin{array}{l}\text { Área pielocalicial } \\
\text { Factor de forma }\end{array}$ & $\begin{array}{c}19,70 \pm 8,32 \\
0,87 \pm 0,07\end{array}$ & $\begin{array}{r}30,19 \pm 11,07 \\
0,88 \pm 0,01\end{array}$ & $\begin{array}{l}p=0,018 \\
p=0,023\end{array}$ \\
\hline Otras variables & Éxito & Fracaso & valor $\boldsymbol{p}$ \\
\hline Función renal & $45,1 \%$ & $40,9 \%$ & 0,625 \\
$\begin{array}{l}\text { Cirugía previa } \\
\text { (n=17) }\end{array}$ & $11(64,7 \%)$ & $6(35,3 \%)$ & \\
$\begin{array}{l}\text { Sin cirugía previa } \\
\text { (n=50) }\end{array}$ & $31(62 \%)$ & $19(38 \%)$ & 0,843 \\
$\begin{array}{l}\text { Con litiasis } \\
\text { (n=13) }\end{array}$ & $(69,3 \%$ & $4(30,7 \%)$ & \\
$\begin{array}{l}\text { Sin litiasis } \\
\text { (n=54) }\end{array}$ & $33(61,1 \%)$ & $1(39,9 \%)$ & \\
$\begin{array}{l}\text { Malformación asociada } \\
\text { (riñón en herradura) } \\
\text { (n=4) }\end{array}$ & 0 & $4(100 \%)$ & \\
\hline
\end{tabular}


un RID. Se consideró criterio de éxito la desaparición de los síntomas y la normalización de la curva de excreción en el RID.

Para conocer la repercusión de la hidronefrosis en el resultado hemos considerado como variables morfológicas el valor del área de la imagen en el urograma con mayor dilatación y el factor de forma de la misma (a mayor esfericidad en la forma el valor se aproxima más a la unidad), obteniéndose estos valores mediante planimetría de forma. Otra variable estudiada fue la función renal estimada en el estudio isotópico.

Como variables cualitativas se consideraron la existencia de cirugía previa, la asociación de litiasis y la coexistencia de malformación renal.

Para el estudio estadístico hemos aplicado el test de la Chi cuadrado para las variables categóricas y la $\mathrm{T}$ de Student para las variables numéricas. La curva de Kaplan-Meier se utilizó para conocer la probabilidad de fracaso en un determinado momento.

\section{RESULTADOS}

En dos casos hubo accidente vascular durante la cirugía que precisó conversión a cirugía abierta, realizándose una nefrectomía en un caso y en otro una pieloplastia tipo Anderson-Hynes. En el postoperatorio la tasa global de complicaciones fue de $15,5 \%$, si bien la mayoría de ellas $(12,9 \%)$ fueron grado II de Clavien (fiebre en 6 casos y sangrado postoperatorio que precisó transfusión en 4 pacientes). En dos casos la complicación requirió cirugía (Clavien III b) por desinserción ureteral y migración del catéter doble $\mathrm{J}$.

El seguimiento medio fue de 149,26 meses (rango: 82-222 meses). De los 77 pacientes 10 se han perdido en el seguimiento a largo plazo. La tasa de éxito ha sido de $73,1 \%, 65,5 \%$ y $63,2 \%$ al año, 5 años y 10 años de seguimiento, respectivamente. La probabilidad de fracaso durante el primer año de seguimiento fue de $26,9 \%$, a los 5 años de $34,5 \%$ y a los 10 años de $36,8 \%$, como expresa la curva de Kaplan-Meier (Fig. 1).

El estudio de las planimetrías de la imagen de la hidronefrosis reveló un área pielocalicial media de $19,70 \pm 8,32 \mathrm{~cm}^{2}$ en los éxitos frente a un área media de $30,19 \pm 11,07 \mathrm{~cm}^{2}$ en los fracasos, $(\mathrm{p}=0,018)$ (Tabla 2). No hubo diferencias con significación estadística entre los valores del factor de forma en los éxitos y en los fracasos $(0,87$ vs $0,88, p=0,135$, respectivamente).

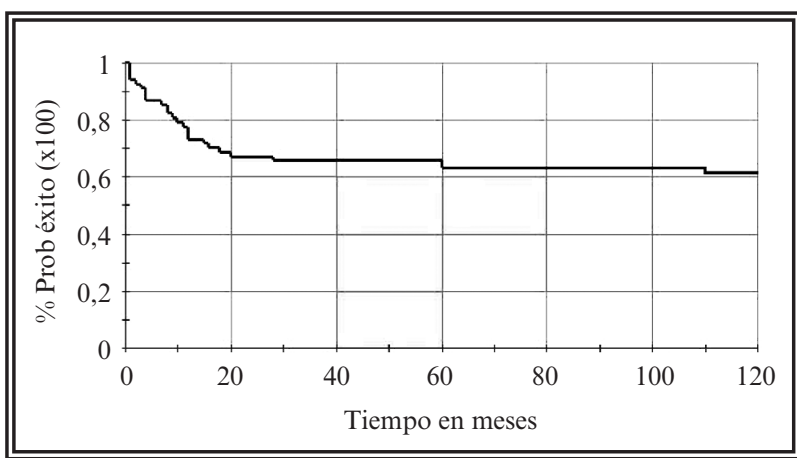

FIGURA 1. Curva de Kaplan-Meier para evaluar la tasa libre de fracaso en función del tiempo.

No apreciamos diferencias significativas en la función renal media de ambos grupos $(45,1 \%$ en éxitos vs $40,9 \%$ en fracasos, $p=0,625$ ), ni en la tasa de éxito entre casos primarios y secundarios $(64,7 \%$ vs $62 \%$, respectivamente, $\mathrm{p}=0,843$ ). Si bien la tasa de éxito para el grupo con OUPU asociada a litiasis fue superior al grupo que presentaba únicamente hidronefrosis $(69,3 \%$ vs $61,1 \%$ respectivamente, $\mathrm{p}=0,541)$, la diferencia no tuvo significación estadística.

En todos los casos en los que la OUPU se asoció a malformación renal (4 casos en riñón en herradura) la endopielotomía fracasó.

\section{DISCUSIÓN}

En los últimos 25 años la endopielotomía se ha consolidado como el tratamiento elección en pacientes adultos con OUPU. Estudios de revisión han aportado tasas de éxito en un rango variable (6589\%) y siempre moderadamente inferiores a la pieloplastia abierta ${ }^{2}$, y han identificado algunos factores anatómicos y funcionales indicativos de fracaso de esta técnica: hidronefrosis severa, función renal pobre y existencia de vaso cruzado. Pero, mientras parece haber consenso en que la función renal mínima debe ser superior al 20\% para obtener un resultado satisfactorio ${ }^{8}$, no se ha cuantificado, hasta la fecha, qué área y morfología de hidronefrosis se relacionan con el fracaso y, por otro lado, existe notable disparidad de opinión al evaluar la importancia del hallazgo de vaso cruzado en la proximidad de la UPU.

Otro aspecto que debe esclarecerse, dada la perspectiva temporal que tenemos de esta técnica, es si existe estabilidad en los resultados con el paso del tiempo. Recientes estudios de revisión de la endopielotomía a largo plazo indican que la tasa de éxito cae significativamente en seguimientos tardíos ${ }^{4-6}$. 
Este inconveniente, junto a la difusión de la pieloplastia laparoscópica, que al reproducir la técnica abierta puede emular sus resultados con las ventajas de su menor morbilidad, cuestiona el papel futuro de la endopielotomía, relegando su indicación al fracaso de aquéllas.

La tasa de éxito de nuestra serie a largo plazo $(63,2 \%)$ es sensiblemente más pobre que la que exponen estudios de revisión (67-87\%) $)^{2,10,11}$. Una explicación plausible a este hecho podría ser el criterio que hemos seguido en la evaluación del resultado. La constatación al inicio del seguimiento de casos de fracaso en la resolución de la obstrucción en el RID, a pesar de existir mejoría clínica en dichos pacientes, nos ha conducido a incorporar el RID tanto en el inicio del seguimiento como tardíamente.

Además, nuestra tasa de éxito ha descendido en el seguimiento tardío si atendemos a los resultados presentados hace 10 años ${ }^{12}$. Pero, si estratificamos esta caída, hemos observado que la mayoría de los fracasos se han producido en los veinte primeros meses del seguimiento. Hemos verificado que la probabilidad de fracaso aumentó un 7,6\% a los cinco años sobre el seguimiento inicial al año, pero solo se incremento un 2,3\% entre los 5 y 10 años siguientes, por lo que podríamos considerar que hubo una cierta estabilidad en los resultados a partir del primer lustro de seguimiento, a diferencia de la caída progresiva y mantenida que refieren los escasos estudios existentes al respecto ${ }^{4,5,6}$.

Parece ser que hay acuerdo en la identificación de la hidronefrosis como uno de los factores más determinantes del fracaso de la endopielotomía ${ }^{8,13}$.

Estratificando los resultados de acuerdo al grado de hidronefrosis, $\mathrm{Lam}^{22}$ comunica una tasa de éxito que varía entre un $66,7 \%$ para las graves y un $100 \%$ para las leves. En nuestro estudio hemos cuantificado el área de la hidronefrosis y lo hemos correlacionado con el resultado, encontrando una marcada diferencia entre el área media en los éxitos y en los fracasos. Además, en un intento de determinar si también la morfología de la hidronefrosis puede ser un factor pronóstico, hemos comprobado que el factor de forma de las cavidades pielocaliciales dilatadas no ha tenido influencia en el resultado. Podríamos, pues, a luz de estos hallazgos, establecer un área pielocalicial máxima, independientemente de la forma, para indicar la endopielotomía con intención de mejorar los resultados.
En nuestro estudio retrospectivo que corresponde al periodo anterior a la introducción de la pieloplastia laparoscópica, no hemos incluido la presencia de vaso cruzado como variable, ya que en ese periodo no realizábamos su detección preoperatoria. Si en un principio se despertó la discusión entre los que desaconsejan la endopielotomía en presencia de vaso cruzado aún en ausencia de gran hidronefrosis $^{14}$ y los que no consideraban que el vaso cruzado fuese un factor decisivo del fracaso ${ }^{16}$, la controversia persiste, además, en la variabilidad tanto de las tasas preoperatorias de vaso polar detectadas por métodos diagnósticos de imagen (44-79\%) ${ }^{8,18}$,como en las tasas de hallazgo de vaso en la pieloplastia primaria (20-42\%) ${ }^{19,20}$. Dado que un $65 \%$ de individuos poseen una arteria polar inferior que cruza la cara anterior de la UPU, según los estudios realizados por Sampaio en cadáver ${ }^{17}$, la endopielotomía obtendría unos resultados mucho mas pobres que los comunicados si la presencia de vaso fuese la única razón de la obstrucción. Seguimos, pues, sin distinguir qué vasos son realmente los causantes de obstrucción y qué vasos son inocentes a pesar de la proximidad a la UPU. Conlin intenta clarificar este problema discriminando mediante ultrasonografía intraoperatoria los casos con vaso > de $4 \mathrm{~mm}$ de diámetro, en los que indica pieloplastia laparoscópica, de los casos con vaso $<4 \mathrm{~mm}$, en los que consigue una tasa de éxito de $70 \%$ con endopielotomía ${ }^{15}$.

Parece claro, además, la asociación entre hidronefrosis severa y vaso cruzado. Van Cangh comunica una tasa de éxito de 54\% en presencia de gran hidronefrosis y sólo del 33\% cuando se asocia además a vaso polar ${ }^{13}$. Nosotros habíamos hallado una asociación de hidronefrosis severa y vaso cruzado en el $85 \%$ de los fracasos durante la pieloplastia secundaria ${ }^{12}$. Por tanto, la selección de pacientes para esta técnica de acuerdo al área de la hidronefrosis puede tener mayor sentido que hacerlo en función de la existencia de vaso cruzado. Los resultados hallados indican que por debajo de $20 \mathrm{~cm}^{2}$ de área pielocalicial la probabilidad de éxito es alta, y por encima de $30 \mathrm{~cm}^{2}$ de área lo es la probabilidad de fracaso. Investigar la existencia de vaso en estos grupos no tendría interés. En cambio, en el grupo intermedio (área pielocalicial: 20-30 $\mathrm{cm}^{2}$ ) la detección de vaso cruzado podría inclinarnos hacia la pieloplastia.

El estudio de la función renal en nuestro trabajo no tuvo relevancia, pues no hubo una diferencia sig- 
nificativa entre la función de ambos grupos, dado que uno de los escasos criterios de selección seguidos para la endopielotomía fue una función renal superior al $20 \%$ como habían señalado otros autores $^{13,16}$

Se han comunicado diferencias en los resultados de la endopielotomía dependiendo de que existiese cirugía previa o fuese un caso primario. Mientras que algunos grupos refieren mejores resultados en las estenosis secundarias ${ }^{13}$ explicando este hecho porque después de una cirugía fracasada la estenosis suele ser pequeña, otros, por el contrario consideran que el carácter isquémico de este tipo de estenosis sería causa de peores resultados ${ }^{23,24}$. En nuestro estudio, si bien la tasa de buenos resultados fue más alta en los casos en los que había fallado la pieloplastia inicial, esta diferencia no tuvo significado estadístico, por lo que, al igual que han encontrado otros autores ${ }^{25}$, la cirugía previa no afectó al resultado, al igual que ocurrió con la concurrencia de litiasis en el paciente.

La incidencia de OUPU en riñón en herradura varia ente un $15 \%$ a un $33 \%$, afectando esta malformación al 0,1-0,25\% de la población ${ }^{27}$, por lo que las series en las que se ha comunicado el empleo de esta técnica con buenos resultados aportan muy pocos $\operatorname{casos}^{26-28}$.

En nuestra serie, aunque han sido solo 4 pacientes en los que la OUPU se acompañaba de esta malformación, (número insuficiente para establecer conclusiones con valor estadístico), el fracaso en todos ellos nos hace pensar que en este tipo de obstrucción influyen otros factores como la malrotación, la inserción alta del uréter, y la existencia del istmo, por lo que la indicación de pieloplastia laparoscópica sería más adecuada.

\section{CONCLUSIÓN}

La tasa de éxito inicial de la endopielotomía disminuye en seguimientos a largo plazo. La mayor concentración de fracasos se produce dentro de los 2 primeros años. A partir de los cinco años de seguimiento los resultados se pueden considerar relativamente estables, por lo que sería aconsejable realizar un seguimiento durante este periodo de tiempo.

Seleccionar casos para esta técnica de acuerdo al área de la hidronefrosis puede mejorar sensiblemente los resultados. La endopielotomía no debería indicarse en hidronefrosis que asienten en riñón en herradura.

\section{REFERENCIAS}

1. Wickham JE, Kellet MJ. Percutaneous pyelolisis. Eur Urol 1983;9(2):122 124.

2. Manikandan R, Saad A, Bhatt RI, Neilson 2. D. Minimally invasive surgery for pelviureteral junction obstruction in adults a critical review of the options. Urology. 2005;65(3)422-432.

3. Schuessler WW, Grune MT, Tecuanhuey IV, Preminger GM. Laparoscopic dismembered pyeloplasty. J Urol. 1993;150(6): 1795-1799.

4. Knudsen, BE CookAJ, Watterson JD, Beiko DT, Nott L, Razvi H Denstedt JD Percutaneous antegrade endopyelotomy: Long-term results from one institutution. Urology. 2004;63:230-234.

5. Doo CK, Hong B, Park T, Park HK. Long-term outcome of endopyelotomy for the treatment of ureteropelvic junction obstruction: How should be patients followed up?. J Endourol. 2007;21(2): 158-161.

6. Dimarco DS, Gettman MT, McGee SM, Chow GK, Leroy AJ, Slezak J, Patterson DE, Segura JW. Long-term success of antegrade endopyelotomy compared with pyeloplasty at a single institution. J Endourol. 2006;20(10) 707-712.

7. Vicente Rodríguez J, Amón Sesmero J H, Estébanez Zarranz J, Martínez Sagarra, JM. Tácticas en riñón: cirugía percutánea. Arch Esp Urol. 2005; 58(8):775-780

8. Kavoussi LR, Albala DM, Clayman R. Outcome of secondary open surgical procedure in patients who failed primary endopyelotomy. Br J Urol. 1993 72(2): 157-160.

9. Gupta M, Tunkay OT, Smith AD. Open surgical exploration after failed endopyelotomy: a 12 years perspective. J Urol. 1997;157(3):1613-1618

10. Muntaz FH, Kommu SS, Siddiqui E, Le Roux PJ, Hellawel G, Hemal AK. Minimally invasive treatment of ureteropelvic junction obstruction: optimizing outcomes with concomitant cost reduction. J Endourol. 2006;20(9):663-668.

11. Van Cangh PJ, Nesa S. Prognostic factors and patient selection. Urol Clin North Am. 1998;25(2):281-288.

12. Amón JH, Estébanez J, Mtez-Sagarra JM. Endopielotomía. ¿Alternativa definitiva a la pieloplastia? Actas Urol Esp. 1993;18:351-358.

13. Van Cangh PJ, Wilmart JF, Opsoner RJ. Long-term results and late recurrence after endoureteropyelotomy: acritical análisis of prognostic factors. J Urol.1994;151(4):934-937.

14. Van Cangh PJ, Nesa S, Galeon M, Tombal B, Dardenne N, Opsomer R, Lorge F. Vessels around ureteropelvic junction: Significance and imaging by conventional radiology. J Endourol. 1996;10:111-119.

15. Conlin MJ. Results of selective management of ureteropelvic junction obstruction. J Endourol 2002;16(2):233-236.

16. Gupta M, Smith AD. Crossing vessels. Endourologic implications. Urol Clin North Am. 1998;25(2):289-293.

17. Sampaio FJ, Favorito LA. Ureteropelvic junction stenosis: vascular anatomies background for endopyelotomy. J Urol 1993 150(6):1787-1791.

18. Janetschek G, Peschel R, Frauscher F. Laparoscopic pyeloplasty. Urol Clin North Am. 2000;27(4):695-704.

19. Mandhani A, Kumar D, Kumar A, Dubey D, Kapoor R. Safety profile and complications of transperitoneal laparoscopic pyeloplasty: a critical analysis. J Endourol. 2005;19(7):797-802.

20. Eden CG, Cahill D, Allen JD. Laparoscopic dismembered pyeloplasty: 50 consecutive cases. BJU Int. 2001;88(6):526-531.

21. Motola JA, Badlani GH, Smith AD. Results of 212 consecutive enpopyelotomies: an 8 year follow-up. J Urol. 1993;149(3):453-456.

22. Korth K, Kuenkel M, Karsch J. Percutaneous endopyelotomy and results: Korth technique. J Endourol. 1996;10(2):121-126.

23. Millán Rodríguez F, Vayreda Martija JM, Segarra Tomás J, Salvador Bayarri $\mathrm{J}$, Vicente Rodriguez Role of percutaneous endopyelotomy in the treatment of pyeloureteral junction stenosis after failed ureteropyeloplasty. J Arch Esp Urol. 1997;50(2):151-158.

24. Kuenkel M, Korth K. Endopyelotomy: long term follow-up of 143 patients. J Endourol. 1990;4:109-115.

25. Hoenig DM, Shalhav AL, Elbanhasy AM, McDougall EM, Smith D, Clayman RV. Impact of etiology of secondary ureteropelvic junction obstruction on outcome of endopyelotomy. J Endourol. 1998;12(2)131-133.

26. Nakamura K, Baba S, Tazaki H. Endopyelotomy in horseshoe kidneys. J Endourol. 1994;8(3):203-206.

27. Bellman GC, Yamaguchi R. Special considerations in endopielotomy in a hor seshoe kidney. Urology. 1996;47(4):582-586.

28. Yohannes $\mathrm{P}$, Smith $\mathrm{AD}$. The endourological management of complication associated with horseshoe kidney. J Urol. 2002;168(1):5-8.

Correspondencia autor: Dr. José H. Amón Sesmero

Servicio de Urología. Hospital Universitario Río Hortega

Rondilla de Sta. Teresa, s/n - 47010 Valladoli. Tel.: 983420400

E-mail autor: amonsesmero@yahoo.es

Información artículo: Original - Endourología

Trabajo recibido: octubre 2008

Trabajo aceptado: diciembre 2008 